Research Article

\title{
Determination of the Strain-Free Configuration of Multispan Cable
}

\author{
Chuancai Zhang, ${ }^{1}$ Qiang Guo, ${ }^{1,2}$ and Xinhua Zhang ${ }^{1}$ \\ ${ }^{1}$ State Key Lab for Mechanical Structural Strength and Vibration, Xian Jiaotong University, Xian, Shannxi 710049, China \\ ${ }^{2}$ Shanghai Electric Power Generation Equipment Co., Ltd. Shanghai Turbine Plant, Shanghai 200240, China \\ Correspondence should be addressed to Xinhua Zhang; xhzhang@mail.xjtu.edu.cn
}

Received 16 March 2015; Revised 25 May 2015; Accepted 26 May 2015

Academic Editor: Tai Thai

Copyright (c) 2015 Chuancai Zhang et al. This is an open access article distributed under the Creative Commons Attribution License, which permits unrestricted use, distribution, and reproduction in any medium, provided the original work is properly cited.

For building a reasonable finite element geometric model, a method is proposed to determine the strain-free configuration of the multispan cable. The geometric conditions (the end conditions and the unstretched length conditions) as constraints for the configuration of multispan cable are given. Additionally, asymptotic static equilibrium conditions are given for determining the asymptotic shape of the multispan cable. By solving these constraint equations, a set of parameters are determined and then the strain-free configuration of multispan cable is determined. The method reported in this paper provides a technique for building reasonable FEA geometric model of multispan cables. Finally, a three-span cable is taken as example to illustrate the effectiveness of the method, and the computed results are validated via the software ADINA.

\section{Introduction}

The cable structures are widely used in the engineering, such as bridge, building, and power transmission system $[1,2]$. In [1], a new force method is proposed for analysing the dynamic behaviour of flat-sag cable structures. The accepted dynamic model of such cables reduces to a partial differential equation and an integral equation. The support reaction forces are considered as excitations, allowing D'Alembert's solution to be used. In this way, single or multispan cables have been developed in the form of a single-degree-of-freedom system in terms of the additional dynamic tension. Finally, two examples are presented to illustrate the accuracy of the proposed force method for single and multispan cable systems subjected to harmonic forces. In [2], a three-dimensional modeling procedure is proposed for cable-stayed bridges with rubber, steel, and lead energy dissipation devices. In this study, the cable structure can be simplified as a fournode isoparametric cable element. The multispan cables and towers are the basic structures in the power transmission system. Under the wind load, the multispan cable is apt to generate large displacements because of its high flexibility and large span. So the multispan cable exhibits strong geometrical nonlinearity. Usually, the dynamic behavior of such cable structures is analyzed on the platform of the finite element analysis (FEA) software. To build a reasonable FEA model, two alternative configurations of the cable structures need to be determined in the modeling process; one is the initial static equilibrium configuration and the other is the assumed strain-free configuration. As a prestressed state, the static equilibrium configuration of a cable is the starting point of the subsequent dynamic analysis, whereas the strain-free configuration of a cable is only an assumed state which does not exist in the real world due to the ubiquitous gravity and it is the starting point for static and dynamic analyses. If the FEA geometric model is built based upon the static equilibrium configuration of the cable, the prestrain or prestress in each element caused by gravity should be specified one element by one element; this is always cumbersome. The gravity load is included in the equilibrium configuration. The FEA geometric model contains two gravity loads in this way, so that the model does not match the actual cable structure. 
The gravity load is not included in the strain-free configuration. Alternately, if the geometric model is built based on the strain-free configuration, the prestrain or prestress in each element needs not to be specified in advance and they are set up naturally in the nonlinear static analysis via the FEA. After that, the gravity load applied in the model; the system achieves equilibrium. Therefore, the assumed strain-free configuration is more preferable to the static equilibrium configuration for building the FEA geometric model of multispan cable.

For the static and dynamic analysis of cable structures, Irvine [3] systematically summarized the classical achievements reached up to 1981 and the static equilibrium shape of a cable is described as an elastic catenary. For the modeling of cable structures, in [4], a method for modeling cable supported bridges for nonlinear finite element analysis is presented in this paper. A two-node catenary cable element, derived using exact analytical expressions for the elastic catenary, is proposed for the modeling of cables. Based upon the elastic catenary, Such et al. [5] proposed a method for determining the static equilibrium configuration of arbitrary three-dimensional cable structures subject to gravity and point loads. Srinil et al. [6, 7] formulated a system of nonlinear partial differential equations describing the large amplitude three-dimensional free vibrations of inclined sagged elastic cables. Ai and Imai [8] proposed an iterative scheme to find the static equilibrium shape of beam-cable mixed system. In [9], based on exact analytical expressions of elastic catenary, Thai and Kim presented a catenary cable element for the nonlinear static and dynamic analysis of cable structures. In [10], Vu et al. presented a spatial catenary cable element for the nonlinear analysis of cable-supported structures and proposed an algorithm for form-finding of cable-supported structures. Rienstra [11] derived the partial differential equations and boundary conditions satisfied by the static equilibrium configuration of multiple spans coupled via suspension strings. In [12], Impollonia et al. obtained the deformed shape of the elastic cable in closed form for the cases of uniformly distributed load and multiple-point forces. Greco and Cuomo [13] proposed a method for obtaining an exact configuration of slack cable nets by using the exact expressions of the equilibrium derived from the equation of the catenary.

All the above papers focused on the determination of static equilibrium configuration of the cable structures. However, the present paper concentrates on determining the strain-free or unstretched configuration of multispan cable. Firstly, based upon the elastic catenary theory, a set of geometric boundary conditions, cable length condition, and static equilibrium conditions are derived for determining the configuration of the multispan cables when they are approaching asymptotically to the strainfree state. These constraint conditions constitute a system of nonlinear algebraic or transcendental function equations. Secondly, these nonlinear equations are solved and one finds a set of parameters which determine the unstretched configuration of the multispan cable. Finally, an example of three-span cable is given to illustrate the method.

\section{The Governing Equations of Multispan Cable}

First we assume that (1) the bending stiffness of cable is negligible; (2) the material of cable is of Hookian type; that is, its constitutive relation is linear elastic; and (3) only the self-weight as the static force is applied to the cable structure. Under these assumptions, the governing equation for the cable in its static equilibrium state is as follows [6]:

$$
\begin{array}{r}
\left(\frac{E A \varepsilon_{0}}{\sqrt{1+y_{0}^{\prime 2}}}\right)^{\prime}=0, \\
\left(\frac{E A \varepsilon_{0} y_{0}^{\prime}}{\sqrt{1+y_{0}^{\prime 2}}}\right)^{\prime}+\frac{\omega_{c} \sqrt{1+y_{0}^{\prime 2}}}{\left(1+\varepsilon_{0}\right)}=0 .
\end{array}
$$

Here, the "'” denotes the derivative with respect to $x, \varepsilon_{0}$ denotes the tensional strain in the cable when it is in static equilibrium state, $E$ is Young's modulus, $A$ is the sectional area of the cable and assumed to be constant, $y_{0}$ represents the static equilibrium shape of the cable, and $\omega_{c}$ is the cable weight per unit length in the unstretched state.

The tensional strain $\varepsilon_{0}$ is related to the horizontal tensional force $T_{H}$ as follows:

$$
\varepsilon_{0}=\frac{T_{H}}{E A} \sqrt{1+y_{0}^{\prime 2}} .
$$

According to (1), the final static equilibrium governing equation of the cable can be rewritten in the following form:

$$
\left(1+\frac{T_{H}}{E A} \sqrt{1+y_{0}^{\prime 2}}\right) y_{0}^{\prime \prime}+\frac{\omega_{c}}{T_{H}} \sqrt{1+y_{0}^{\prime 2}}=0 .
$$

This is the so-called elastic catenary equation. Being different from the traditional catenary equation, it takes account of the extensibility of the cable. Under the dynamic wind load, the multispan cable usually vibrates nonlinearly due to its large displacement, so the finite element analysis is preferable to investigate the static and dynamic behavior. To build the FEA model, firstly the geometric model of multispan cable is needed. Therefore, one of the initial configurations, the strain-free state or the static equilibrium state, should be determined. As stated in the Introduction, in the following, we only discuss how to determine the strain-free configuration of the multispan cable.

\section{Determination of the Strain-Free Configuration}

Determining the strain-free configuration of the cable based on the data of static equilibrium configuration can be viewed as an inverse problem in nonlinear structural mechanics. The unstretched state of cable is an assumed state and it can be viewed as an asymptotic state that corresponds to $E A \rightarrow \infty$ (which means the cable is inextensible), 


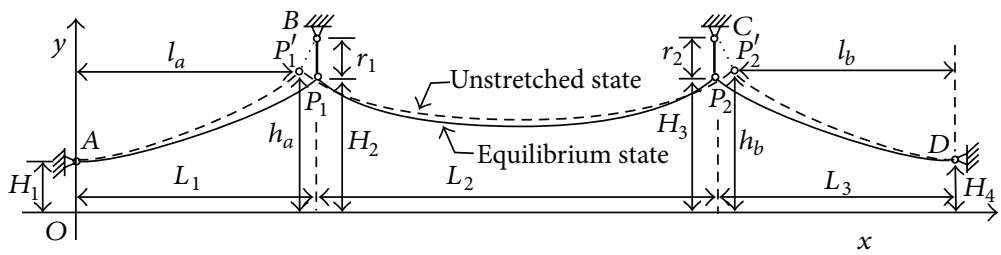

FIgURE 1: The configuration of three-span cable.

and $\omega_{c} / T_{H} \rightarrow-a$ (note that $\omega_{c}$ is negative), so the equation for cable in unstretched state is as follows:

$$
y^{\prime \prime}-a \sqrt{1+y^{\prime 2}}=0 .
$$

This is the rigid catenary equation. Its solution can be found as

$$
y(x)=\frac{1}{a} \cosh a\left(x+c_{1}\right)+c_{2} .
$$

For the three-span cable, as illustrated in Figure 1, the shape of the left span cable in its unstretched state can be expressed as

$$
y_{1}(x)=\frac{1}{a_{1}} \cosh a_{1}\left(x+c_{11}\right)+c_{12} .
$$

Similarly, the shape of the middle span cable can be expressed as

$$
y_{2}(x)=\frac{1}{a_{2}} \cosh a_{2}\left(x+c_{21}\right)+c_{22}
$$

and the shape of the right span cable is expressed as

$$
y_{3}(x)=\frac{1}{a_{3}} \cosh a_{3}\left(x+c_{31}\right)+c_{32} .
$$

There are 9 unknown parameters appeared in the above expressions. Determination of the strain-free configuration of the three-span cable is equivalent to determining these unknown parameters, so the following constraint equations can be built.

For the left span cable,

$$
\text { End conditions: }\left\{\begin{array}{l}
\left.y_{1}(x)\right|_{x=0}=H_{1}, \\
\left.y_{1}(x)\right|_{x=l_{a}}=h_{a}, \\
\left(L_{1}-l_{a}\right)^{2}+\left(H_{2}+r_{1}-h_{a}\right)^{2}=r_{1}^{2} .
\end{array}\right.
$$

Here, the meaning of the first and second equations of (9) is obvious. In engineering, the $\operatorname{rod} A P_{1}$ (insulator) is very stiff so it can be reasonably assumed as rigid; therefore, the third equation of (9) holds too. Figure 1 can be referred to for the meaning of the symbols $L_{1}, H_{1}, H_{2}, l_{a}, h_{a}$, and $r_{1}$ in the above equations. In addition, the following condition about the length of the original unstretched cable can be built:

$$
\text { Length condition: } \int_{0}^{l_{a}} \sqrt{1+y_{1}^{\prime 2}} d x=s_{01} \text {. }
$$

Here, $s_{01}$ represents the length of the left span cable in its unstretched state and can be expressed via the data of the static equilibrium configuration.

It should be pointed out that, in some cases (e.g., in the example given later), the original length $s_{01}$ of the cable in its unstretched state may be less than the straight distance $A P_{1}$, so, in order to build a reasonable FEA geometric model, the position of the hanging point $P_{1}^{\prime}$ must be determined.

Note that the function $y_{1}(x)$ is in the form of cosh; the above integral actually can be given explicitly. To find the length $s_{01}$, the following equations can be constructed (see [3], page 18):

$$
\begin{aligned}
& \frac{T_{H} s_{01}}{E A}+\frac{T_{H} s_{01}}{W_{1}}\left\{\sinh ^{-1}\left(\frac{V_{1}}{T_{H}}\right)\right. \\
& \left.-\sinh ^{-1}\left(\frac{V_{1}-W_{1}}{T_{H}}\right)\right\}=L_{1}, \\
& \frac{W_{1} s_{01}}{E A}\left(\frac{V_{1}}{W_{1}}-\frac{1}{2}\right)+\frac{T_{H} s_{01}}{W_{1}}\left[\left\{1+\left(\frac{V_{1}}{T_{H}}\right)^{2}\right\}^{1 / 2}\right. \\
& \left.-\left\{1+\left(\frac{V_{1}-W_{1}}{T_{H}}\right)^{2}\right\}^{1 / 2}\right]=H_{1}-H_{2},
\end{aligned}
$$

where $W_{1}\left(=\omega_{c} s_{01}\right)$ is the self-weight of the left span cable and $T_{H}$ and $V_{1}$ are the horizontal and vertical reaction forces at the support point $A$, respectively. From the above two equations, the unknown $V_{1}$ and $s_{01}$ can be solved.

Being similar to (9), for the middle and right span cables, the following constraint equations can also be constructed. For the middle span cable,

$$
\text { End conditions: }\left\{\begin{array}{l}
\left.y_{2}(x)\right|_{x=l_{a}}=h_{a}, \\
\left.y_{2}(x)\right|_{x=L_{1}+L_{2}+L_{3}-l_{b}}=h_{b}, \\
\left(L_{3}-l_{b}\right)^{2}+\left(H_{3}+r_{2}-h_{b}\right)^{2}=r_{2}^{2},
\end{array}\right.
$$

Length condition: $\int_{l_{a}}^{L_{1}+L_{2}+L_{3}-l_{b}} \sqrt{1+y_{2}^{\prime 2}} d x=s_{02}$.

For the right span cable,

$$
\text { End conditions: }\left\{\begin{array}{l}
\left.y_{3}(x)\right|_{x=L_{1}+L_{2}+L_{3}-l_{b}}=h_{b}, \\
\left.y_{3}(x)\right|_{x=L_{1}+L_{2}+L_{3}}=H_{4},
\end{array}\right.
$$

Length condition: $\int_{L_{1}+L_{2}+L_{3}-l_{b}}^{L_{1}+L_{2}+L_{3}} \sqrt{1+y_{3}^{\prime 2}} d x=s_{03}$. 
Again, Figure 1 can be referred to for the meaning of the symbols $H_{3}, H_{4}, L_{2}, L_{3}, l_{b}, h_{b}$, and $r_{2}$ in the above equations. $s_{02}$ and $s_{03}$ represent, respectively, the lengths of middle and right span cables in their unstretched state.

Similar to (11), the determining equations for $s_{02}$ are as follows:

$$
\begin{aligned}
& \frac{T_{H} s_{02}}{E A}+\frac{T_{H} s_{02}}{W_{2}}\left\{\sinh ^{-1}\left(\frac{V_{2}}{T_{H}}\right)\right. \\
& \left.-\sinh ^{-1}\left(\frac{V_{2}-W_{2}}{T_{H}}\right)\right\}=L_{2}, \\
& \frac{W_{2} s_{02}}{E A}\left(\frac{V_{2}}{W_{2}}-\frac{1}{2}\right)+\frac{T_{H} s_{02}}{W_{2}}\left[\left\{1+\left(\frac{V_{2}}{T_{H}}\right)^{2}\right\}^{1 / 2}\right. \\
& \left.-\left\{1+\left(\frac{V_{2}-W_{2}}{T_{H}}\right)^{2}\right\}^{1 / 2}\right]=H_{3}-H_{2}
\end{aligned}
$$

and the equations for $s_{03}$ are as follows:

$$
\begin{aligned}
& \frac{T_{H} s_{03}}{E A}+\frac{T_{H} s_{03}}{W_{3}}\left\{\sinh ^{-1}\left(\frac{V_{3}}{T_{H}}\right)\right. \\
& \left.-\sinh ^{-1}\left(\frac{V_{3}-W_{3}}{T_{H}}\right)\right\}=L_{3}, \\
& \frac{W_{3} s_{03}}{E A}\left(\frac{V_{3}}{W_{3}}-\frac{1}{2}\right)+\frac{T_{H} s_{03}}{W_{3}}\left[\left\{1+\left(\frac{V_{3}}{T_{H}}\right)^{2}\right\}^{1 / 2}\right. \\
& \left.-\left\{1+\left(\frac{V_{3}-W_{3}}{T_{H}}\right)^{2}\right\}^{1 / 2}\right]=H_{3}-H_{4} .
\end{aligned}
$$

Besides (9), (10), and (12)-(14), two other equations describing the equilibrium conditions of forces in the limiting state from $P_{1} \rightarrow P_{1}^{\prime}$ and $P_{2} \rightarrow P_{2}^{\prime}$ are given as follows:

$$
\text { At point } P:\left(\mathbf{T}_{1}+\mathbf{T}_{2}\right) \cdot \boldsymbol{\tau}=0 \text {. }
$$

Referring to Figure 2, the unnormalized directional vector $\mathbf{n}$ can be written in its coordinate-component form as

$$
\mathbf{n}=\left(l_{a}-L_{1}, h_{a}-\left(H_{2}+l_{1}\right)\right)
$$

and the unnormalized directional vector $\boldsymbol{\tau}$ can also be given in its coordinate-component form as

$$
\boldsymbol{\tau}=\left(h_{a}-\left(H_{2}+l_{1}\right), L_{1}-l_{a}\right) .
$$

The tensional forces $\mathbf{T}_{1}$ and $\mathbf{T}_{2}$ in the left span and middle span cables can be written in their component form as

$$
\begin{aligned}
& \mathbf{T}_{1}=-\left.\left\|\mathbf{T}_{1}\right\|\left(\frac{1}{\sqrt{1+y_{1}^{\prime 2}}}, \frac{y_{1}^{\prime}}{\sqrt{1+y_{1}^{\prime 2}}}\right)\right|_{x=X_{P_{1}^{\prime}}}, \\
& \mathbf{T}_{2}=-\left.\left\|\mathbf{T}_{2}\right\|\left(\frac{1}{\sqrt{1+y_{2}^{\prime 2}}}, \frac{y_{2}^{\prime}}{\sqrt{1+y_{2}^{\prime 2}}}\right)\right|_{x=X_{P_{1}^{\prime}}} .
\end{aligned}
$$

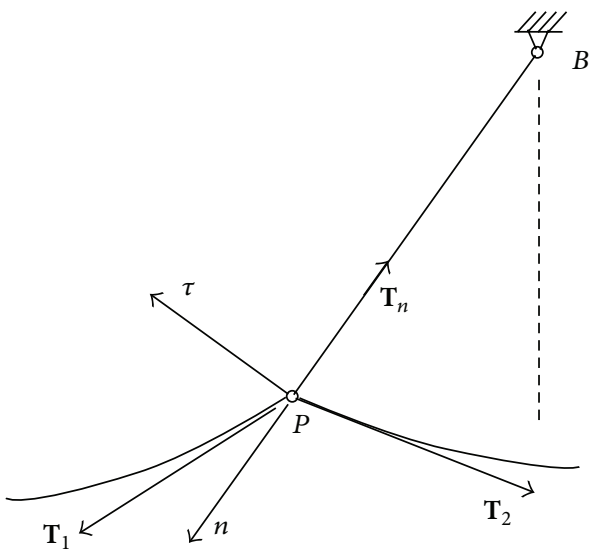

Figure 2: The forces at the suspension point.

On the other hand, in the limit case $\left(\omega_{c} \rightarrow 0\right)$, the following relations hold:

$$
\begin{aligned}
& \frac{\omega_{c}}{\left\|\mathbf{T}_{1}\right\| / \sqrt{1+y_{1}^{\prime 2}}}=-a_{1}, \\
& \frac{\omega_{c}}{\left\|\mathbf{T}_{2}\right\| / \sqrt{1+y_{2}^{\prime 2}}}=-a_{2} .
\end{aligned}
$$

Substituting these relations into (13), one finally gets

$$
\begin{aligned}
\left(\frac{1}{a_{2}}\right. & \left.-\frac{1}{a_{1}}\right)\left(H_{2}+l_{1}-h_{a}\right) \\
& +\left.\left(l_{a}-L_{1}\right)\left(\frac{y_{2}^{\prime}}{a_{2}}-\frac{y_{1}^{\prime}}{a_{1}}\right)\right|_{x=l_{a}}=0 .
\end{aligned}
$$

Similar to (22), at point $C$ the following relation holds:

$$
\begin{aligned}
& \left(\frac{1}{a_{3}}-\frac{1}{a_{2}}\right)\left(H_{3}+l_{2}-h_{b}\right) \\
& \quad+\left.\left(L_{3}-l_{b}\right)\left(\frac{y_{3}^{\prime}}{a_{3}}-\frac{y_{2}^{\prime}}{a_{2}}\right)\right|_{x=L_{1}+L_{2}+L_{3}-l_{b}}=0 .
\end{aligned}
$$

Theoretically, the strain-free configurations of cable structures with any number of spans can be determined in the same way as described above. On the other hand, there are infinity of many strain-free configurations for the multispan cables; each of them satisfies the geometric constraints, that is, the end conditions and the length conditions. By imposing the force equilibrium conditions, we obtain actually the asymptotic strain-free configuration of the cable. In a sense, such an asymptotic configuration (from the static equilibrium state to the strain-free state) is optimal, because it is most similar to the static equilibrium shape of the cable, so if one performs the FEA starting from this configuration, the convergence is expected to be most rapid.

For determining the configuration of the three-span cable in its unstretched state, totally, there are 13 unknowns including $a_{i}, c_{i j}(i=1 \cdots 3, j=1,2)$ and $h_{a}, h_{b}, l_{a}$, and $l_{b}$ 
TABLE 1: The parameters of three-span cable.

\begin{tabular}{lc}
\hline Parameter & Value \\
\hline$H_{1}$ & 40 \\
$H_{2}$ & 206 \\
$H_{3}$ & 210 \\
$H_{4}$ & 45 \\
$L_{1}$ & 580 \\
$L_{2}$ & 1910 \\
$L_{3}$ & 570 \\
$T_{H}$ & 91378 \\
$E$ & $6.3 e+10$ \\
$A$ & $6.6655 e-4$ \\
$\rho$ & 4524.1 \\
$\omega_{c}$ & 29.0668 \\
\hline
\end{tabular}

TABLE 2: The solution of three-span cable.

\begin{tabular}{lc}
\hline Parameter & Value \\
\hline$a_{1}$ & $0.2591 e-3$ \\
$a_{2}$ & $0.2791 e-3$ \\
$a_{3}$ & $0.2590 e-3$ \\
$c_{11}$ & 802.4259 \\
$c_{12}$ & -3903.9257 \\
$c_{21}$ & -1527.5928 \\
$c_{22}$ & -3502.7267 \\
$c_{31}$ & 3879.8891 \\
$c_{32}$ & -3903.5631 \\
$h_{a}$ & 206.0680 \\
$h_{b}$ & 210.0671 \\
$l_{a}$ & 578.8362 \\
$l_{b}$ & 1912.3203 \\
\hline
\end{tabular}

and 13 equations including (9)-(10), (12)-(14), and (22)-(23). Because of the complex nonlinearity, these equations can only be solved numerically. In the next section, an engineering example is given as illustration.

\section{An Example}

To illustrate the proposed method for determining the strainfree configuration of multispan cable, in this section a real three-span transmission line system is taken as example; its data are listed as shown in Table 1.

Substituting these data into the above equations and then using the Newton-Raphson method, one can find a set of solutions as shown in Table 2.

The unstretched lengths of the three span cables are found to be $s_{01}=602.7090463, s_{02}=1935.110206$, and $s_{03}=592.8032497$, respectively. Knowing the values of these parameters, the asymptotic configuration of the three-span cable in its strain-free state can be described by (6)-(8).

Via the above computed results, the original unstretched configuration of the three-span cable can be easily plotted as shown in Figure 3. Starting from this configuration, the

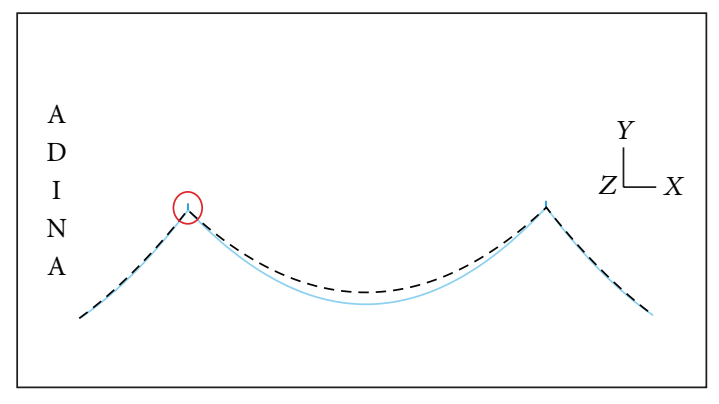

- - - Unstretched configuration

- Static equilibrium configuration

FIGURE 3: The unstretched and the static equilibrium configurations of the three-span cable.

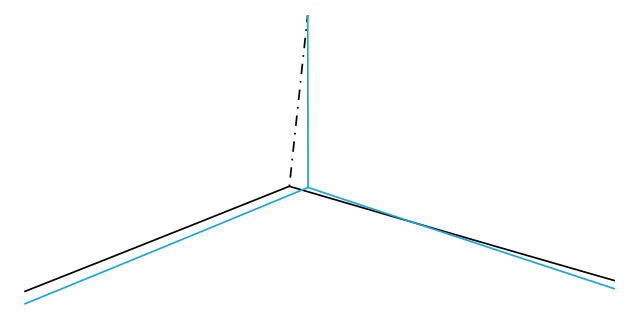

FIgURE 4: The magnification of local configuration near the left insulator.

geometric model for FEA can be easily built, and the corresponding static equilibrium configuration can be further determined by using FEA software, for example, the ADINA. The static equilibrium configuration via the ADINA is also shown in the same figure. The largest difference in height between the unstretched and the static equilibrium configurations is about $17.75 \mathrm{~m}$.

On the other hand, to see clearly the difference between the original unstretched and the static equilibrium configurations, a zoom-in of the local configurations of the three-span cable near the left insulator is shown in Figure 4.

\section{Conclusions}

In this work, a method is presented for determining the asymptotic strain-free configuration of the multispan cable. The constraints of the geometric conditions and the asymptotic static equilibrium conditions are derived, and the determining equations for the asymptotic strain-free configuration are obtained. A set of parameters which describe the shape of the multispan cable are determined. The proposed method provides a way to build reasonable FEA geometric model of multispan cables. At last, a three-span cable system is taken as example to illustrate the effectiveness of the method; the results are validated via the ADINA.

\section{Conflict of Interests}

The authors declare that there is no conflict of interests regarding the publication of this paper. 


\section{References}

[1] X. Ma and J. W. Butterworth, "A force method model for dynamic analysis of flat-sag cable structures," Shock and Vibration, vol. 16, no. 6, pp. 623-635, 2009.

[2] H. M. Ali and A. M. Abdel-Ghaffar, "Seismic passive control of cable-stayed bridges," Shock and Vibration, vol. 2, no. 4, pp. 259272, 1995.

[3] H. M. Irvine, Cable Structure, MIT Press, Boston, Mass, USA, 1981.

[4] R. Karoumi, "Some modeling aspects in the nonlinear finite element analysis of cable supported bridges," Computers and Structures, vol. 71, no. 4, pp. 397-412, 1999.

[5] M. Such, J. R. Jimenez-Octavio, A. Carnicero, and O. LopezGarcia, "An approach based on the catenary equation to deal with static analysis of three dimensional cable structures," Engineering Structures, vol. 31, no. 9, pp. 2162-2170, 2009.

[6] N. Srinil, G. Rega, and S. Chucheepsakul, "Large amplitude three-dimensional free vibrations of inclined sagged elastic cables," Nonlinear Dynamics, vol. 33, no. 2, pp. 129-154, 2003.

[7] N. Srinil, G. Rega, and S. Chucheepsakul, “Three-dimensional non-linear coupling and dynamic tension in the largeamplitude free vibrations of arbitrarily sagged cables," Journal of Sound and Vibration, vol. 269, no. 3-5, pp. 823-852, 2004.

[8] M. Ai and H. Imai, "A shape-finding analysis of suspended structures on the displacement-method equilibrium," Structural Engineering/Earthquake Engineering, vol. 21, no. 1, pp. 27-41, 2004.

[9] H.-T. Thai and S.-E. Kim, "Nonlinear static and dynamic analysis of cable structures," Finite Elements in Analysis and Design, vol. 47, no. 3, pp. 237-246, 2011.

[10] T.-V. Vu, H.-E. Lee, and Q.-T. Bui, "Nonlinear analysis of cablesupported structures with a spatial catenary cable element," Structural Engineering and Mechanics, vol. 43, no. 5, pp. 583605, 2012.

[11] W. Rienstra, "Nonlinear free vibrations of coupled spans of suspended cables," in Proceedings of the 3rd European Conference on Mathematics in Industry, J. Manley, S. McKee, and D. R. Owens, Eds., pp. 133-144, 1988.

[12] N. Impollonia, G. Ricciardi, and F. Saitta, "Statics of elastic cables under 3D point forces," International Journal of Solids and Structures, vol. 48, no. 9, pp. 1268-1276, 2011.

[13] L. Greco and M. Cuomo, "On the force density method for slack cable nets," International Journal of Solids and Structures, vol. 49, no. 13, pp. 1526-1540, 2012. 

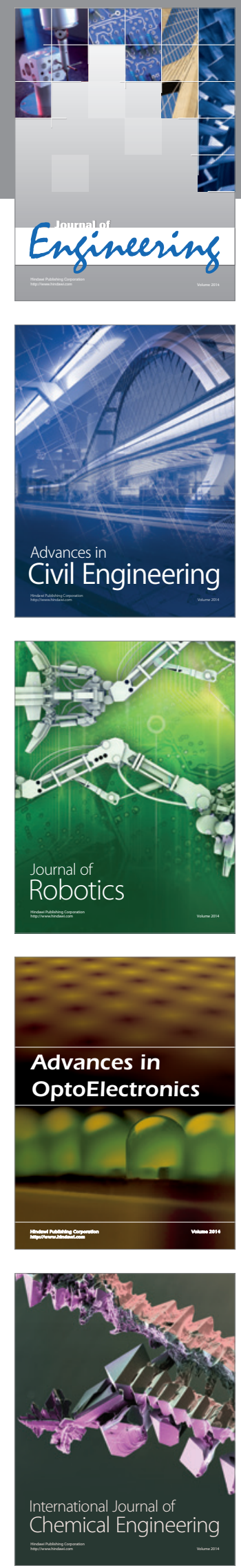

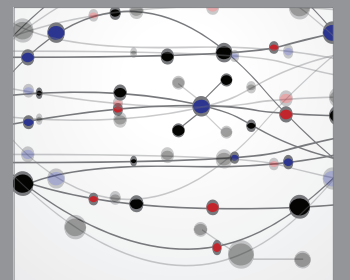

The Scientific World Journal
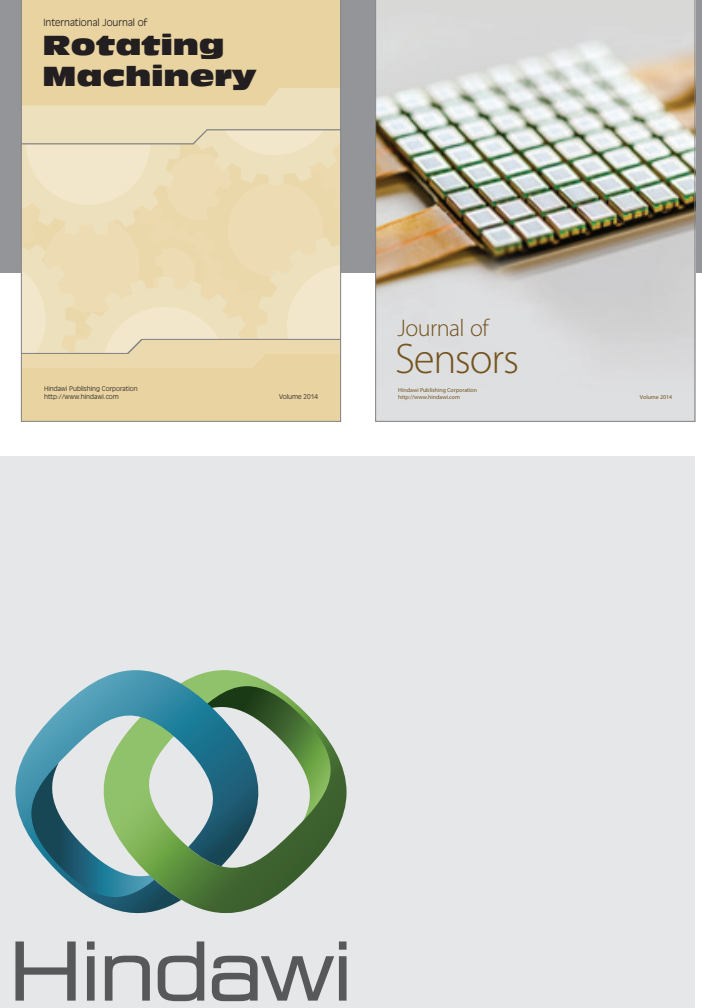

Submit your manuscripts at http://www.hindawi.com
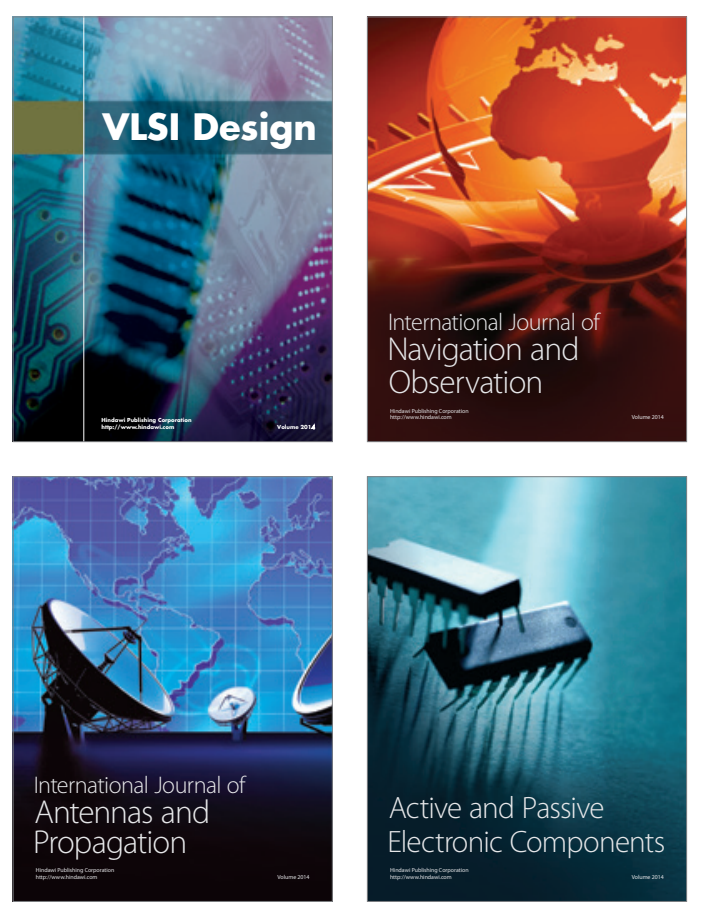
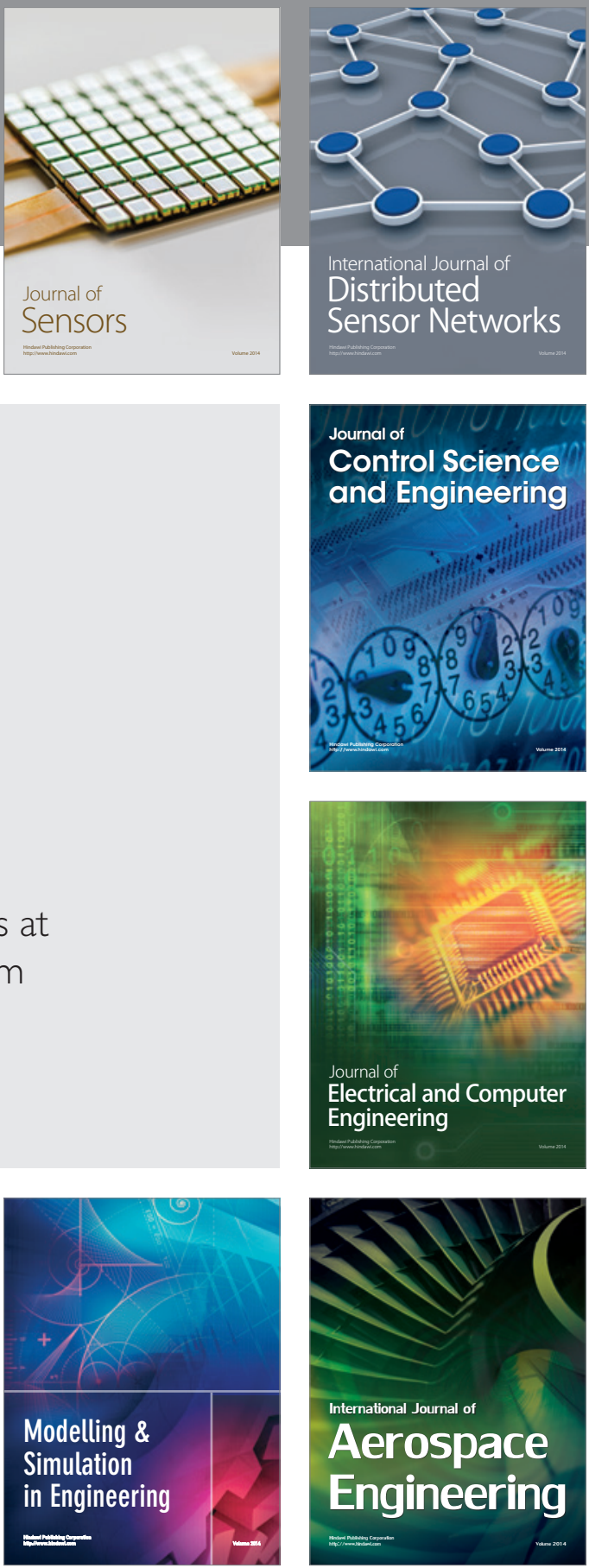

Journal of

Control Science

and Engineering
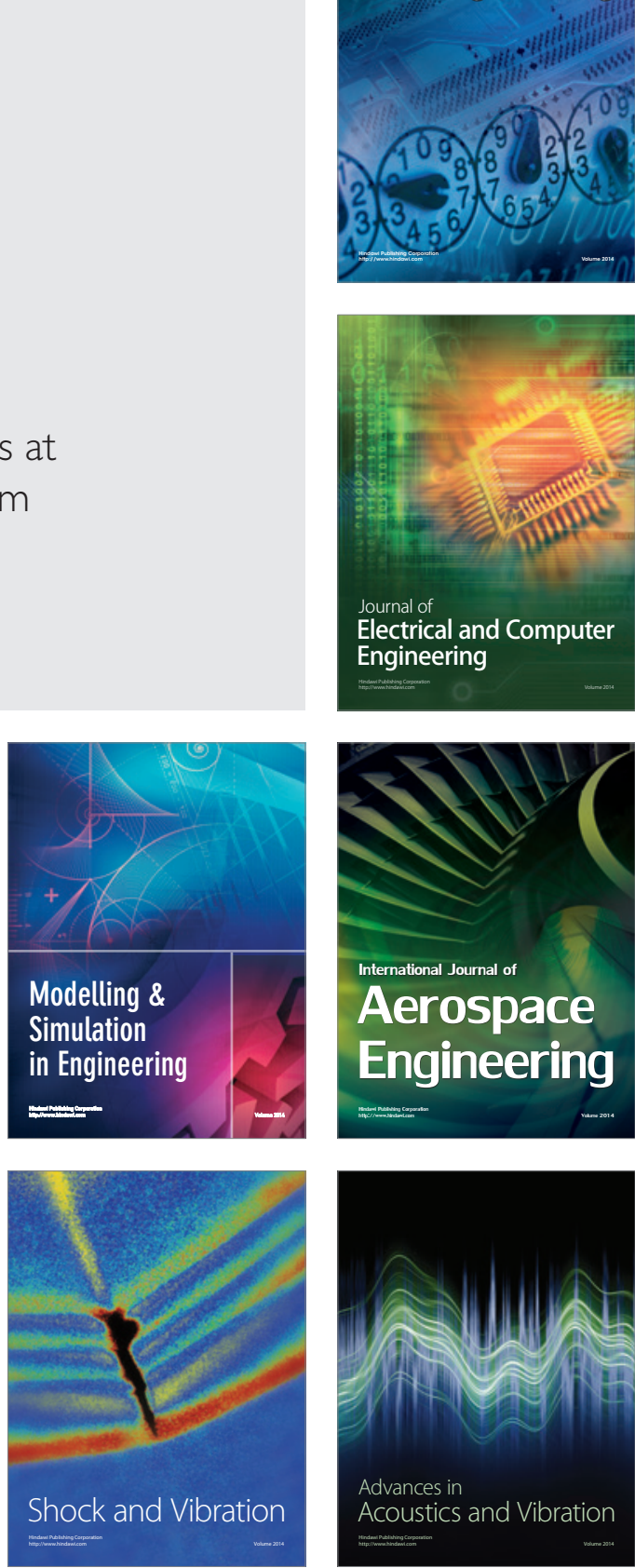